\section{Polar science: global partnership to work on data sharing}

SIR - Your Editorial 'The way ahead for polar science' (Nature 457, 1057; 2009) correctly points out the critical need for a sustained archive of the data collected during International Polar Year (IPY). As you mention in your News Feature 'In from the cold' (Nature 457, 1072-1077; 2009), "the IPY

data-management committee is sorting out the options for setting up a fully integrated data-sharing system". The primary mechanism for this data sharing is the International Polar Year Data and Information Service (IPYDIS).

The IPYDIS is a global partnership of data centres, archives and networks, which together ensure proper stewardship of IPY and related data (http://ipydis.org). We seek to create a union catalogue that provides access to all IPY data distributed in archives around the world.

As you emphasize, sustained support for this activity is a critical challenge for nations and for the international science community. Australia, Canada, China, the Netherlands, Norway, Russia, Sweden, the United Kingdom, the United States and others have already started setting up a system, but the work to present the IPY data legacy has only just begun.

Although the IPY data policy encourages more comprehensive and more timely release of data, data sharing is still a challenge for polar science. We need to build on our success and extend the effort to a broader community. To this end, the International Council for Science has established a new project, 'The Polar Information Commons: establishing the framework for long-term stewardship of polar data and information'. The council's committee on data for science and technology, CODATA, leads this initiative to establish a sustainable long-term framework for the preservation of and access to polar data.

We encourage a 'commons' approach, as used in other disciplines, that recognizes information as a common societal benefit. Historically, society has managed both to share and administer common property, such as fisheries and telecommunications bandwidth, but to sustain these resources requires ongoing societal attention. This approach can strengthen incentives for scientists, research institutions and nations to contribute and document data, reduce barriers to data sharing and provide a focal point for data assessment by the community.

Mark A. Parsons National Snow and Ice Data Center/World Data Center for Glaciology, University of Colorado, 449 UCB, Boulder, Colorado 80309-0449, USA

e-mail:parsonsm@nsidc.org

\section{Polar science: bid for freely accessible biodiversity archive}

SIR - The Southern Ocean is of unique ecological, biogeographic and political interest, as noted by your Editorial 'The way ahead for polar science' (Nature 457, 1057; 2009). Given the rate and potential catastrophic impact of global change on the Antarctic marine ecosystem, we agree that it is vital to have a mechanism for the exchange of relevant scientific information. Information about Antarctic marine biodiversity has to be widely published, instantly accessible and thoroughly checked, in order to enable timely, science-based management.

An example of the kind of initiative you call for is the Marine Biodiversity Information Network of the Scientific Committee on Antarctic Research (SCAR-MarBIN). Since 2005, the network's web portal has compiled and managed information on Antarctic marine biodiversity (www. scarmarbin.be). It is home to the first complete Register of Antarctic Marine Species, a fully operable, browsable and searchable online species list, maintained by more than 70 expert taxonomic editors.

The register offers access to information on more than 15,000 taxa and contributes to the World Register of Marine Species and to the Catalogue of Life.

Through a mapping system, SCAR-MarBIN also allows baseline data on marine organisms to be visualized and downloaded. This offers, for the first time, a way to quantify the diversity and distribution of Antarctic marine life and to measure how, when and where it has been studied. This information is also made available through larger initiatives, such as the Ocean Biogeographic Information System or the Global Biodiversity Information Facility.

The data published in SCARMarBIN constitute a benchmark for detecting responses to global change, enabling the scientific community to highlight key areas that require investigation or protection. Currently focusing on biogeographic data, SCARMarBIN is developing tools to improve its usefulness for conservation and sustainable management.

As part of the legacy of International Polar Year, various initiatives are collaborating to document biodiversity in the Southern Ocean, the ecosystem services it delivers and its evolution in the light of global change. Creating a freely accessible archive should be considered a top priority, with funding from all the International Polar Year nations relative to their economic strength. It is a matter of responsibility towards future generations.

If they are successful, initiatives including SCAR-MarBIN will safeguard the legacy of these efforts, helping to preserve Antarctica as was designated by the Antarctic Treaty: as "a natural reserve, devoted to peace and science".

Bruno Danis Royal Belgian Institute of Natural Sciences, 29 rue Vautier, 1000 Brussels, Belgium e-mail: bruno.danis@scarmarbin.be Huw Griffiths British Antarctic Survey, Natural Environment Research Council, Cambridge CB3 OET, UK

\section{Canadian government reaffirms support for science and discovery}

SIR - You report researchers' concerns about the Canadian government's support for science in two recent News stories (Nature 457, 646; 2009 and Nature 458, 393; 2009). As Minister of State for Science and Technology, I can say that, despite the global economic situation, the government of Canada remains committed to innovation and discovery. We have increased funding to researchers, both in universities and in the private sector.

In the past three years, for example, we have significantly increased the budgets of federal granting councils, increased scholarships through the Canada Graduate Scholarships Program, and increased the Industrial Research Assistance Program for small and medium-sized businesses. The Budget 2009 announcements include Can $\$ 750$ million (US $\$ 590$ million) for the Canada Foundation for Innovation to attract and retain world-leading researchers, and a Can\$2-billion infrastructure programme. The government has also put in place two five-year funding agreements with Genome Canada that are worth Can $\$ 240$ million, to support large-scale, world-class research.

Your readers should therefore rest assured that the government of Canada will continue to fund research for the benefit of all scientists and Canadians.

Gary Goodyear Industry Canada, 235 Queen Street, Ottawa, Ontario K1A OH5, Canada e-mail: gary.goodyear@ic.gc.ca 